\title{
CRESCIMENTO E PRODUÇÃO DE AFLATOXINAS POR Aspergillus flavus $\mathrm{E}$ Aspergillus parasiticus
}

\author{
MARIA MARLUCIA GOMES PEREIRA * \\ ELIANA PINHEIRO DE CARVALHO ** \\ GUILHERME PRADO ***
}

\begin{abstract}
Efetuou-se revisão de literatura sobre as condições de crescimento dos fungos toxigênicos Aspergillus flavus e Aspergillus parasiticus em alimentos. Foram abordados os principais fatores que determinam o crescimento de fungos e a produção de aflatoxina (atividade de água, temperatura, potencial hidrogênico, potencial de oxirredução, composição do substrato e aditivos). Concluiu-se que a presença do fungo no alimento não implica, obrigatoriamente, em produção de micotoxina, assim como a toxina pode estar presente no alimento mesmo na ausência do fungo. A contaminação de alimentos por fungos significa perdas econômicas e risco à saúde do consumidor.
\end{abstract}

PALAVRAS-CHAVE: FUNGOS; MICOTOXINAS.

\section{INTRODUÇÃO}

Durante muito tempo os fungos foram considerados vegetais. A partir de 1960 passaram a ser classificados como reino à parte - Fungi. Os fungos são seres vivos eucarióticos unicelulares como as leveduras, ou pluricelulares como os fungos filamentosos ou bolores e os cogumelos. Os fungos não sintetizam clorofila nem qualquer pigmento fotossintético (43).

* Professora Assistente, Departamento de Morfofisiologia Veterinária, Centro de Ciências Agrárias, Universidade Federal do Piauí, Teresina, PI, Doutoranda, Departamento de Ciência dos Alimentos, Universidade Federal de Lavras. (e-mail: marlug@ufla.br).

** Professor Adjunto, Departamento de Ciência dos Alimentos, Universidade Federal de Lavras, Lavras, MG.

*** Farmacêutico Bioquímico, Pesquisador Pleno 3, Núcleo de Micologia e Micotoxinas, Fundação Ezequiel Dias, Belo Horizonte, MG. 
Os esporos dos fungos são abundantes e amplamente encontrados na natureza, germinam rapidamente no solo, em plantas, em alimentos, em papel e até em vidros. Os alimentos armazenados representam excelente campo para a proliferação dos fungos, principalmente quando os princípios básicos de secagem adequada e armazenamento correto são desconhecidos ou desprezados (11).

As substâncias denominadas aflatoxinas são produzidas por espécies de fungos, essencialmente por Aspergillus flavus e Aspergillus parasiticus. O gênero Aspergillus pertence ao grupo dos Hyphomycetos que se caracteriza pela formação de conidióforos, ou seja, hifas especializadas e produtoras de conídios com formas e arquitetura variáveis $(10,28)$.

A contaminação e a deterioração dos alimentos causadas por fungos são mais comuns que as originadas por qualquer outro grupo de microrganismos. A contaminação por fungos é importante não apenas sob o ponto de vista sensorial, mas também pelo perigo que a produção de micotoxinas representa para o consumidor (23).

Os fungos podem promover prejuízos significativos aos alimentos. Quando presentes em sementes ocasionam perda do poder germinativo. No arroz e na manteiga de cacau afetam a qualidade, promovendo descoloração, e no café produzem aromas desagradáveis. Podem, ainda, alterar as condições físicas dos produtos, reduzir o valor nutritivo, alterar o aspecto externo, produzir aflatoxinas e favorecer a ação de outros agentes de deterioração, como leveduras, bactérias e insetos (11).

Os trópicos estão situados na parte do globo entre as latitudes de aproximadamente $23^{\circ}$ norte e $23^{\circ}$ sul. Nem todas as áreas compreendidas nessa faixa são quentes e úmidas, mas em sua maioria as condições atmosféricas são muito favoráveis (70 a 100\% de umidade e mais de $25^{\circ} \mathrm{C}$ ) para rápida proliferação dos fungos (11).

A pesquisa de aflatoxina teve início em 1960, na Inglaterra, quando ocorreu intoxicação de aves tratadas com rações à base de amendoim provenientes do Brasil e da África. Tal fato ficou conhecido como "Doença X" e, na época, causou a morte de milhares de animais (20).

A aflatoxina tem recebido grande atenção em comparação com as demais micotoxinas, devido aos efeitos carcinogênicos que podem provocar em animais e o efeito agudo tóxico em seres humanos. As aflatoxinas representam o grupo de micotoxinas com mais resultados positivos em 
alimentos já relatados $(44,37)$.

Em termos de maior e menor susceptibilidade, a contaminação dos alimentos por fungos (12), tendo em vista sua natureza, composição e uso, ocorre na seguinte ordem: cereais, subprodutos de cereais (essencialmente o trigo), subprodutos de matadouros de aves, farinhas de alfafa, mandioca, soja integral, girassol integral, algodão, farinha de soja, farinha de girassol, glúten de arroz e outros, além de produtos submetidos a peletização.

O objetivo geral desta revisão consistiu em levantar dados sobre as condições de crescimento dos fungos toxigênicos A. flavus e A.parasiticus em alimentos e sua importância em saúde pública.

\section{FATORES IMPORTANTES PARA O CRESCIMENTO DE FUNGOS E PRODUÇÃO DE AFLATOXINA}

Os principais fatores que determinam a deterioração dos alimentos são a atividade de água, a concentração hidrogeniônica, a temperatura (processamento e estocagem), o potencial de oxirredução, a consistência, os nutrientes, o efeito específico do soluto e os preservativos (28).

\subsection{ATIVIDADE DE ÁGUA}

A produção de aflatoxina resulta da tripla combinação de espécie fúngica, do substrato e do meio ambiente. Os fatores que afetam a produção de aflatoxina podem ser divididos em 3 categorias, ou seja, física, nutricional e biológica (14).

A atividade de água requerida para o crescimento do $A$. flavus é de 0,80 a >0,99 (ótimo 0,98). Para a produção da aflatoxina os valores são de 0,82 a >0,99 e a atividade de água considerada mais favorável é de 0,95 a $0,99(15)$.

A umidade relativa de equilíbrio corresponde à umidade de que dispõem os microrganismos, uma vez alcançados o equilíbrio entre a umidade livre do produto e o vapor de água existente no meio ambiente. A umidade relativa de equilíbrio é expressa em porcentagem e varia de alimento para alimento, pois depende de sua composição em glicídios e gorduras. De forma geral, a umidade relativa de equilíbrio refere-se à atmosfera em 
equilíbrio com o produto e a atividade de água do próprio produto $(12,28)$.

PITT e MISCAMBLE (29) avaliaram o crescimento de $A$. flavus, $A$. parasiticus e A. nomius, em temperaturas de 25,30 e $37^{\circ} \mathrm{C}$ e diferentes atividades de água (entre 0,75 a 0,996). Foram utilizadas glicose e frutose para o controle da atividade de água no meio, sendo que a relação atividade de água entre as espécies mostrou-se similar. O mínimo de atividade de água para germinação e crescimento das espécies estudadas foi de 0,82 a $25^{\circ} \mathrm{C}, 0,81$ a $30^{\circ} \mathrm{C}$ e 0,80 a $37^{\circ} \mathrm{C}$. O Aspergillus nomius foi ligeiramente menos xerofílico que as demais espécies.

A umidade do substrato e a umidade relativa constituem pontos críticos na produção da aflatoxina. De acordo com observações de DIENER e DAVIS (7) a produção máxima de aflatoxina, em grão de cereal, ocorre em umidade de $25 \%$ a $30^{\circ} \mathrm{C}$. A umidade relativa mínima exigida é de 83 a $88 \%$. Tais autores observaram, também, aumento na produção de aflatoxina com o acréscimo da umidade relativa para $99 \%$.

A influência da atividade de água foi analisada em amendoim, variedade "Tatu vermelho", quanto ao crescimento de fungos e à produção de aflatoxina. Observou-se que nos primeiros 15 dias em atividade de água de 0,75 a 0,86 o crescimento do fungo foi lento, sendo mantido o mesmo nível durante 60 dias, em todas as atividades de água testadas $(0,75$; 0,$83 ; 0,86 ; 0,93$ e 0,97 ). A produção ótima de aflatoxina ocorreu em atividade de água de 0,93 e, em 0,86 não houve formação de aflatoxina por 120 dias (30).

Amostras de arroz com microbiota fúngica natural ou amostra estéril inoculada com uma espécie de fungo ou misto de fungos ( $A$. flavus, A. niger, P. purpurogenum e F. moniliforme) foram estocadas em diferentes concentrações de umidade. O tempo inicial para moldagem da população fúngica e a produção de aflatoxina $\mathrm{B} 1$ foram avaliados. A população fúngica do arroz naturalmente contaminado e com umidade de $13 \%$ diminuiu significativamente durante a estocagem. Com 17 e $20 \%$ de umidade ocorreu aumento, atingindo o máximo de $\log _{10}{ }^{7}\left(\mathrm{UFCg}^{-1}\right)$. As amostras com umidade maior ou igual a $20 \%$ apresentaram níveis elevados (>20 ppb) de aflatoxina B1. A síntese de aflatoxina em umidade de $25 \%$ aumentou a incorporação de maior quantidade de inóculo de $A$. flavus, não ocorrendo o mesmo quando da utilização de miscelânea de fungos. O inóculo de $A$. flavus e $P$. purpurogenum apresentou baixa produção de aflatoxina e a síntese máxima ocorreu apenas mediante o inóculo de $A$. flavus (25). 


\subsection{TEMPERATURA}

Em alimentos refrigerados e não-refrigerados, estocados em residências, foi observado que a microbiota fúngica era representada por Penicillium (49\%) e por Aspergillus (38\%). Destes, 10,7\% foram capazes de produzir micotoxinas, incluindo aflatoxinas, ácido cójico, ocratoxina $A$, ácido penicílico e patulina. Verificou-se, ainda, que a produção de aflatoxina foi mais comum nos alimentos não-refrigerados (42).

A temperatura ótima para a produção de aflatoxina é influenciada pelo tipo de substrato. No amendoim, em solução nutriente, a temperatura ótima para a produção de aflatoxina por Aspergillus flavus foi $25^{\circ} \mathrm{C}$, em período de incubação de 7 a 9 dias. Elevados índices de aflatoxina foram produzidos em amendoim a 25 e $30^{\circ} \mathrm{C}$, durante 7 e 15 dias de incubação, ocorrendo o mesmo no meio líquido. A produção de aflatoxina por Aspegillus parasiticus atingiu maior nível nas temperaturas de 20, 25 e $30^{\circ} \mathrm{C}$, no período de 5 a 21 dias. Os níveis mais elevados foram observados aos 25 e $30^{\circ} \mathrm{C}$, com 7 e 15 dias de incubação (7).

Para SORENSON et al. (40) a temperatura ótima para a produção de aflatoxina em arroz foi de $28^{\circ} \mathrm{C}$, não sendo detectada a toxina em temperatura abaixo de $8{ }^{\circ} \mathrm{C}$ ou a partir de $37^{\circ} \mathrm{C}$. A temperatura ótima para a produção de aflatoxina geralmente encontra-se entre 25 a $28^{\circ} \mathrm{C}(13)$.

TORREY e MARTH (42) avaliaram o crescimento de uma cepa de Aspergillus produtora de aflatoxina, na temperatura de $8^{\circ} \mathrm{C}$, durante 504 horas e não observaram crescimento durante este período.

De acordo com o ICMSF (15) os limites de crescimento do Aspergillus flavus variam de 10 a $43^{\circ} \mathrm{C}$ e a temperatura considerada ótima é de $33^{\circ} \mathrm{C}$. A temperatura para a produção de aflatoxina é de 13 a $37^{\circ} \mathrm{C}$, tendo como ótimo temperaturas entre 16 a $31^{\circ} \mathrm{C}$. Para o Aspergillus parasiticus a temperatura varia de 12 a $42^{\circ} \mathrm{C}$, tendo como temperatura mais favorável $32^{\circ} \mathrm{C}$. Já a produção da aflatoxina ocorre entre 12 a $42^{\circ} \mathrm{C}$ e a temperatura considerada ótima é de $25^{\circ} \mathrm{C}$.

A produção de aflatoxina foi analisada em várias temperaturas pelo período de 5 dias em meio Wort. A produção máxima de aflatoxina foi observada a $24^{\circ} \mathrm{C}$ e o crescimento máximo do $A$. flavus ocorreu nas temperaturas de 29 e $35^{\circ} \mathrm{C}$. Durante 5 dias não houve produção de aflatoxina em temperatura abaixo de $18^{\circ} \mathrm{C}$ e acima de $35^{\circ} \mathrm{C}(36)$. 
A estabilidade da aflatoxina no leite e seus derivados têm sido estudada e os resultados causado discordância entre os pesquisadores. EGMOND (8) estudou leite naturalmente contaminado, submetido à esterilização e à pasteurização, não observando redução na concentração de AFM1. Resultado idêntico foi obtido por STOLOFF et al. (41), que não encontraram redução da AFM em amostras de leite contaminadas artificial e naturalmente e pasteurizadas a $62^{\circ} \mathrm{C}$ por 30 minutos, e a $77^{\circ} \mathrm{C}$ por 16 segundos, respectivamente. Já os resultados encontrados por PURCHASE e STEYN (32) demonstraram que no leite pasteurizado a $62{ }^{\circ} \mathrm{C}$ por 30 minutos houve redução de $32 \%$ na concentração da $A F M 1$. A redução foi de 45 e $64 \%$ quando o leite foi submetido a temperaturas de 72 e $80^{\circ} \mathrm{C}$ por 45 segundos, respectivamente. A estabilidade da aflatoxina em condições de resfriamento e congelamento também variou. Em amostras de leite cru, naturalmente contaminadas e armazenadas a $0^{\circ} \mathrm{C}$ por 4 e 6 dias, a redução na concentração mostrou-se rápida, com perdas de $40 \%$ e $80 \%$, respectivamente. Em amostras armazenadas a $-18^{\circ} \mathrm{C}$ por 120 dias, a diminuição na concentração ocorreu mais lentamente, com perdas de $14 \%$ aos 30 dias e $86 \%$ aos 120 dias (22).

O efeito do congelamento em amostras naturalmente contaminadas com AFM1 e armazenadas a $-18^{\circ} \mathrm{C}$ apresentou baixa degradação e o processo ocorreu lentamente. Os resultados demonstraram que aos 53 dias não houve redução, sendo verificada pequena variação aos 68 dias. Aos 120 dias houve perda de $45 \%$ da AFM1 (41).

A influência da temperatura e da atividade de água foi avaliada quanto à produção de aflatoxina por $A$. flavus em feijão cowpea, farinha e farinha com cebola. Temperaturas de 21 e $30^{\circ} \mathrm{C}$ produziram elevada quantidade de aflatoxina, sendo a maior quantidade obtida em farinha contendo cebola, com atividade de água de 0,98 e incubação a $21^{\circ} \mathrm{C}$, após 20 dias (19).

Amostras de arroz inoculadas com cepas de $A$. flavus toxigênico foram incubadas a $15^{\circ} \mathrm{C}, 25^{\circ} \mathrm{C}$ e $40^{\circ} \mathrm{C}$ em umidade relativa (UR) entre $61 \%$ e $99 \%$, pelo período de 10, 20 e 30 dias. A melhor produção de aflatoxina foi obtida a $25^{\circ} \mathrm{C}$, em UR de $85 \%$ e 98\%, após 10 dias da incubação (2).

Pipoca branca e amarela foram estocadas em diferentes recipientes, a $35 \stackrel{\circ}{ } \mathrm{C}$ e umidade de $85 \%$ por 3 meses. A infecção interna por fungos no início da estocagem foi baixa em ambas, sendo 5,7\% na pipoca branca e $3 \%$ na pipoca amarela. Pouca diferença foi encontrada no nível de infecção total aos 60 dias de armazenamento, exceto para a espécie $A$. glaucus, a qual predominou em relação às demais. Aumento gradual na infecção 
fúngica ocorreu durante os 30 dias de estocagem. Houve variação no crescimento dos fungos nos diferentes recipientes. A capacidade dos fungos toxigênicos invadirem as pipocas foi determinada usando inóculos, os quais incluíam as espécies $A$. flavus, $P$. martensi e $P$. viridicatum. No entanto, nenhuma espécie inoculada afetou as amostras durante a estocagem (18).

\subsection{POTENCIAL HIDROGENIÔNICO}

Para BUCHANAN e AYRES (4) o efeito do pH depende da composição do meio. Assim, o pH inicial ótimo para a produção da aflatoxina será determinado pelo meio que será empregado para o crescimento do fungo. Ainda em relação ao potencial hidrogeniônico $(\mathrm{pH})$, maior produção de aflatoxina foi observada em $\mathrm{pH}$ entre 4 e 6 (14). Sabe-se que $\mathrm{pH}$ menor que 6 favorece a produção da AFB1 e AFB2 e pH maior que 6 , a produção de $A F G_{1}$ e $A F G_{2}$.

$\mathrm{O}$ pH ótimo para o desenvolvimento de $A$. flavus e $A$. parasiticus situa-se entre 5 e 8, mas os mesmos podem crescer em ampla faixa de $\mathrm{pH}(2 \mathrm{a}$ $>11$ ). $\mathrm{O} \mathrm{pH}$ para produção de aflatoxina por $A$. parasiticus varia de $2 \mathrm{a}>8$, sendo 6 o pH mais favorável (15).

\subsection{POTENCIAL DE OXIRREDUÇÃO}

O grau de oxigenação assume importante papel no crescimento dos fungos e na produção da aflatoxina, pois ambos são processos aeróbicos (17). A produção de aflatoxina é inibida mediante aumento gradual da concentração de dióxido de carbono de 20 para 100\% (20). O crescimento de fungos e a produção de aflatoxinas são inibidos em concentração de $\mathrm{CO}_{2}$ de 40 a $60 \%$, a $25^{\circ} \mathrm{C}$ e $86 \%$ de UR. Em concentração de $20 \%$ de $\mathrm{CO}_{2}$ ocorre o crescimento do fungo, porém não há formação da micotoxina. Em concentrações de $100 \%$ de $\mathrm{CO}_{2}$ ocorre total inibição do crescimento do fungo, bem como da formação da micotoxina (37).

Amendoim comestível foi submetido a aquecimento de $210^{\circ} \mathrm{C}$ em fluxo contínuo de $\mathrm{N}_{2}, \mathrm{CO}_{2}$ e $\mathrm{O}_{2}$ a 1,3 L/min, assim como amostra controle sem aeração, durante $0,10,18$ e 25 minutos. Foi observado que significância maior ou menor em relação à mudança de cor ocorreu em atmosfera de $\mathrm{O}_{2}$ e $\mathrm{CO}_{2}$, respectivamente. Melhor sabor apresentou o amendoim assado por 18 minutos em atmosfera de $\mathrm{N}_{2}$ e $\mathrm{CO}_{2}$. A redução total dos conteúdos 
de $\propto$-amino nitrogênio, glicose, sacarose, conarachin e atividade lipoxigenase dependeu da extensão do tratamento térmico (6).

O crescimento de $A$. flavus, a produção de aflatoxina e de ácidos graxos livres foram avaliados pela presença de dióxido de carbono em combinação com umidade relativa e temperatura. O fungo desenvolveu-se nas seguintes condições: umidade $86 \%, \mathrm{CO}_{2}-20 \%$ e temperatura $27^{\circ} \mathrm{C}$ e/ou umidade $60 \%, \mathrm{CO}_{2}-40 \%$ e temperatura de $25^{\circ} \mathrm{C}$, mas a produção de aflatoxina e ácidos graxos livres foi inibida. Foi observado, ainda, que os níveis de aflatoxinas e ácidos graxos livres diminuíram com a redução da umidade relativa de $99 \%$ para $92 \%$ e $86 \%$ (35).

O requerimento de oxigênio e as condições antioxidantes de cepas de $A$. parasiticus toxigênicos e não-toxigênicos para a produção de aflatoxinas foram avaliados. As exigências de oxigênio das cepas não-toxigênicas permaneceram praticamente inalteradas durante as várias fases de crescimento. As cepas toxigênicas apresentaram acentuada demanda de oxigênio na fase relativa à produção de aflatoxina (16).

\subsection{COMPOSIÇÃO DO SUBSTRATO}

O meio de cultura Aspergillus Diferencial (ADM) para rápida detecção das espécies aflatoxigênicas de Aspergillus flavus e $A$. parasiticus foi descrito por BOTHAST e FENNELL (3). O componente essencial deste meio é o citrato férrico, o qual promove intensa coloração amarelo-laranja na parte inversa da colônia.

O experimento realizado por PITT et al. (27) demonstrou que, no meio ADM, dois compostos foram essenciais para produção da cor (importante fator na identificação do fungo): nitrogênio orgânico e íons férricos. A fonte de íons férricos utilizada foi o citrato férrico amoniacal a 0,05\% e a de nitrogênio orgânico, o extrato de levedura (3).

Extrato de levedura usado como fonte de nitrogênio orgânico mostrou-se mais efetivo na produção da cor do fungo que a triptona. No entanto, a triptona pode substituir a peptona bacteriológica, pois apresenta menor custo e eficiência idêntica à da peptona (27).

O teor de proteína e de carboidrato constitui importante fator na produção de fungos e micotoxinas. O A. parasiticus, mediante elevado teor de proteínas e baixo teor de carboidratos, não produz altos níveis de aflatoxina, 
embora possa ocorrer extensiva produção de fungos e esporulados. Já o Aspergillus flavus, submetido a baixos teores de carboidratos, é capaz de produzir quantidade substancial de aflatoxina (26).

Elevado rendimento na produção de aflatoxina por $A$. parasiticus, em meio extrato de levedura, aconteceu quando a sacarose foi usada como carboidrato. Moderada produção foi verificada diante dos carboidratos glicose, maltose e frutose. No entanto, a lactose foi incapaz de promover a formação da aflatoxina (9). A produção máxima de aflatoxina ocorreu no meio com $30 \%$ de glicose e o crescimento máximo em $10 \%$ de glicose (38). Para LUCHESE e HARRIGAN (21) glicose e frutose são excelentes fontes de crescimento, esporulação e produção de aflatoxina, tanto para A. flavus quanto para A. parasiticus.

O catabolismo dos carboidratos mostra-se valioso na regulação da síntese de aflatoxina. O Aspergillus no meio PMS (peptona, sal mineral) não produz aflatoxina, no entanto, pode-se observar síntese de aflatoxina ao transferi-lo para o meio GMS (glicose, sal mineral), ou ainda se o meio PMS for acrescido de glicose (5).

A melhor fórmula para a rápida detecção de Aspergillus flavus e Aspergillus parasiticus é composta por extrato de levedura, $20 \mathrm{~g}$; peptona bacteriológica, $10 \mathrm{~g}$; citrato férrico amoniacal, 0,5 g; cloranfenicol, 0,1 g; dicloran - solução estoque (0,2\% em etanol), $1 \mathrm{~mL}$; ágar, $15 \mathrm{~g}$; e água destilada, $1 \mathrm{~L}$. Este meio, denominado Ágar A. flavus e parasiticus (AFPA), pode ser autoclavado e a incubação deve ocorrer a $30^{\circ} \mathrm{C} \pm 1^{\circ} \mathrm{C}$ por 42h (27).

A interação entre microrganismos demonstra que a presença de outros microrganismos, seja bactéria ou outro fungo, altera o crescimento de fungos e a produção de micotoxinas. Os fungos, como as bactérias, competem entre si por nutrientes disponíveis, fato que pode influenciar a produção de micotoxinas (24).

Para AHREMED e HOBSON (1), em condições experimentais, os níveis de aflatoxina em extrato dependem da quantidade dos carboidratos glicose e frutose em solução. Os mesmos testaram extratos de 12 variedades de tamareira (Phoenix dactylifera $L$.) e observaram que todas as variedades foram capazes de propiciar o crescimento do micélio e a síntese de aflatoxina. Verificaram o crescimento de fungo toxigênico como $A$. parasiticus na superfície de extratos de frutas e afirmaram que pode ocorrer também a síntese de aflatoxina. 


\subsection{ADITIVOS}

Os efeitos de diferentes concentrações de ácido acético e propiônico, em meio extrato de levedura-glicose e sal, em pH 4,5 e 5,5 foram avaliados quanto ao crescimento de Aspergillus flavus NRRL 2999 e à produção de aflatoxina. As concentrações máximas de ácido acético e ácido propiônico que permitiram crescimento em pH inicial de 5,5 foram 1\%, após 7 dias de incubação, e $0,25 \%$ depois de 3 dias de incubação, respectivamente. No mesmo meio, com pH inicial de 4,5 , as concentrações máximas de ácido acético ou propiônico que permitiram o crescimento do fungo foram $0,25 \%$ e $0,1 \%$, respectivamente. Em relação à quantidade de micélio (peso seco) não houve diferença significativa $(p>0,05)$ quanto às concentrações de $0,0 \%, 0,25 \%, 0,50 \%$ e $0,75 \%$ de ácido acético. A produção de aflatoxina $\mathrm{B}_{1}$ e $\mathrm{G}_{1}$ diminuiu com o aumento da concentração dos ácidos acético e propiônico (34).

O crescimento de Aspergillus flavus em meio líquido SMKY, com diferentes concentrações de cloreto de sódio e pH 4,5, mostrou que o aumento da salinidade exerceu efeito sobre o crescimento e pronunciado efeito inibitório em relação à produção de aflatoxina (39).

PREVIDI (31) avaliou o crescimento do Aspergillus flavus e a síntese de aflatoxina em meio contendo sacarose e meio extrato de carne, acrescidos de cloreto de sódio (de 0 a 12\%) e incubação de $5,15,25^{\circ} \mathrm{C}$. No meio de cultura com sacarose, a quantidade de aflatoxina produzida a $25^{\circ} \mathrm{C}$ foi significativa em todas as concentrações de sal. Na temperatura de $15^{\circ} \mathrm{C}$ houve crescimento do fungo em todas as concentrações de sal. A produção da aflatoxina ocorreu até $8 \%$ de sal, sendo a maior produção a $2 \%$. Na temperatura de $5{ }^{\circ} \mathrm{C}$ o fungo cresceu, no entanto, não houve produção de aflatoxina. No meio extrato de carne, a quantidade de aflatoxina a $25^{\circ} \mathrm{C}$ foi mil vezes menor que no meio com sacarose. A $15^{\circ} \mathrm{C}$, tanto a redução do crescimento do fungo quanto à síntese da aflatoxina foram consideráveis. Na temperatura de $5^{\circ} \mathrm{C}$ não houve crescimento do fungo em nenhuma concentração salina.

A utilização de sulfato de cálcio ( $\mathrm{CaSO} 4)$ em diferentes concentrações, como suplemento em uma plantação de amendoim foi avaliada. Depois da colheita, os amendoins foram inoculados com $1 \times 10^{7}$ de $A$. parasiticus (NRRL5139) e as amostras mantidas a $25^{\circ} \mathrm{C}$ por 14 dias. A avaliação da biomassa do fungo e a produção da aflatoxina revelaram redução em ambas (33). 


\section{CONCLUSÃO}

A contaminação dos alimentos por fungos significa perdas econômicas consideráveis. Além disso, os fungos toxigênicos podem representar risco à saúde do consumidor, seja a longo ou em curto prazo. A presença do fungo no alimento não implica, obrigatoriamente, em produção de micotoxina, assim como a toxina pode estar presente no alimento mesmo na ausência do fungo. Tanto para o crescimento do fungo quanto para a produção da toxina são necessárias condições especiais que favoreçam seu desenvolvimento e, por conseguinte, a produção da toxina.

\section{Abstract}

GROWING AND PRODUCTION OF AFLATOXINS BY Aspergillus flavus AND Aspergillus parasiticus

A literature review about the growing conditions of the toxigenic fungi Aspergillus flavus and Aspergillus parasiticus in food was made. The main factors that determine the fungal growth and the production of aflatoxin (water activity, temperature, hidrogenic potential, oxi-reduction potential, substrate composition and additives) were studied. It was concluded that the presence of the fungi in food doesn't apply in the production of micotoxin, as well as the toxin may be present even in the absence of the fungi. The contamination of food by fungi denote economical losses and risk to consumers health.

KEY WORDS: FUNGI; MICOTOXINS.

\section{REFERÊNCIAS}

1 AHREMED, I. A.; ROBSON, R. K. The ability of date extracts to support the production of aflatoxins. Food Chemistry, v. 66, p. 307-312, 1999.

2 ASEVEDO, I.G et al. Influence of temperature and relative humidity in the production of aflatoxins in samples of stored maize, artificially contamined with Aspergillus flavus (link). R. Microbiol., São Paulo, v. 24, n. 1, p. 32-37, 1993.

3 BOTHAST, R. J.; FENNEL, D. I. A medium for rapid identification and enumeration of Aspergillus flavus and related organisms. Mycologia, v. 66, p.365-369, 1974.

4 BUCHANAN JR, R. L.; AYRES, J. C. Effect of initial pH on aflatoxin production. Applied Microbiology, v. 30, n. 6, p.1050-1051, Dec. 1975. 
5 BUCHANAN, R. L.; LEWIS, D. F. Regulation of aflatoxin biosynthesis: effect of glucose on activities of various glycolytic enzymes. Applied and Environmental Microbiology, v. 48, n. 2, p. 306-310, Aug. 1984.

6 CHIOU, R. Y. Y.; TSENG, C. Y.; SAPPHIRINE, H. Characteristics of peanut kernels roasted under various atmospheric environments. Journal of Agricultural and Food Chemistry, v. 39, p. 1852-1856, 1991.

7 DIENER, U. H.; DAVIS, N. D. Aflatoxin production by isolates of Aspergillus flavus. Phytopathology, v. 56, p. 1390-1393, 1966.

8 EGMOND, H. P. van. Mycotoxins in dairy products. Food Chem., v. 11, p.289-307, 1983.

9 ENGEL, G. Production of mycotoxins and their quantitative determination 4. Extent of aflatoxin synthesis by Aspergillus flavus in dependence on the quality and quantity of carbohydrates available. Milchwissenschaft, v. 30, p. 588-591, 1975.

10 FIGUEIREDO, M. B. Noções sobre fungos e classificação taxonômica. Disponível: <http: //www.geocities.com/ esabio/ mario/nocoesbasicas.htm>. Acesso em 11 de fevereiro de 2001.

11 FONSECA, H. Os fungos e a deterioração de alimentos. Disponível: <http: //www.micotoxinas.com.br/boletim4.htm>. Acesso em 05 de maio 2000.

12 GIMENO, A. Revisión genérica del problema de los hongos y de las micotoxinas en la alimentación animal. Disponível: <http: //www.micotoxinas.com.br/boletim4.htm>. Acesso em 2 abr.2000.

13 GOURAMA, H.; BULLERMAN, L. B. Aspergillus flavus and Aspergillus parasiticus: aflatoxigenic fungi of concern in foods and feeds: a review. J. Food Protect., v. 58, n.12, p. 13951404, Dez. 1995a.

14 GOURAMA, H.; BULLERMAN, L. B. Detection of molds in foods and feeds: potential rapid and selective methods. J. Food Protect, v. 58, n.12, p. 1389-1394, Dec. 1995b. 
15 ICMSF. Microorganismos de los alimentos: características de los patógenos microbianos. Zaragoza: Acribia, 1996. p. 403428.

16 JAYASHREE, T.; SUBRSMANYAM, C. Oxidative stress as a prerequisite for aflatoxin production by Aspergillus parasiticus. Free Radical Biology \& Medicine, v. 29, n. 10, p. 981-985, 2000.

17 JARVIS, B. Factors affecting the production of mycotoxins. J. Appl. Bact., v. 34, n. 1, p. 199-213, 1971.

18 KATTA, S. K.; BULLERMAN, L. B. Effects of high temperature and relative humidity on mold content and quality of stored popcorn. J. Food Protect., v. 58, n. 9, p. 1018-1022, 1995.

19 KOEHLER, P. E.; BEUCHAT, L. R.; CHHINNAN, M. S. Influence of temperature and water activity on aflatoxin production by Aspergillus flavus in cowpea (Vigna unguiculata) seeds and meal. J. Food Protect., v. 48, n. 12, p. 1040-1043, 1985.

20 LANDERS, K. E., DAVIS, N. D., DIENER, U. L. Influence of atmosfheric gases on aflatoxin production by Aspergillus flavus in peanuts. Phytopathology, v. 57, p. 1086-1090, 1967.

21 LUCHESE, R. H., HARRIGAN, W. F. Biosynthesis of aflatoxinthe role of nutritional factors. J. Appl. Bact., v. 74, p. 5-14, 1993.

22 McKINNEY, J. D. et al. Effects of ammoniation on aflatoxins in rations fed lactating cows. J. Am. Oil. Chem. Soc., Champaign, v. 50 , p. $79-84,1973$.

23 MUNINBAZI, C.; BULLERMAN, L. Molds and mycotoxins in foods from Burundi. J. Food Protect., v. 59, n. 8, p. 869-875, 1996.

24 OMINSKI, K. H. et al. Ecological aspects of growth and mycotoxin production by storage fungi: mycotoxin in grain: compounds other than aflatoxin. [New York]: Miller e Trenholm, 1994. Cap. 6, p. 287-312. 
25 OYEBANJI, A. O.; EFIUVWERE, B. Growth of spoilage mould and aflatoxin $\mathrm{B}_{1}$ production in naturally contaminated or artificially inoculated maize as influenced by moisture content under ambient tropical condition. International Biodeterioration \& Biodegradation, v. 44, p. 209-217, 1999.

26 PARK, K-Y.; BULLERMAN, L. Effects of substrate and temperature on aflatoxin production by Aspergillus parasiticus and Aspergillus flavus. J. Food Protect., v. 46, n. 3, p. 178184, Mar., 1983.

27 PITT, J. I.; HOCKING, A. D.; CSIRO G. D. R. An improved medium for the detection of Aspergillus flavus and A. parasiticus. J. Appl. Bact. v.54, p. 109-114, 1983.

28 PITT, J. I.; HOCKING, A. D. Fungi and food spoilage. $2^{\text {nd }}$ ed. London: Chapman \& Hall, 1997. 593 p.

29 PITT, J. I.; MISCAMBLE, B. F. Water relations of Aspergillus flavus and closely related species. J. Food Protect., v. 58, n. 1, p. 86-90, 1994.

30 PRADO, G. et al. Efeito da umidade relativa na contaminação microbiana e produção de aflatoxinas em amendoim em grão. Ciência Tecnol. Aliment., v.11, n. 2, p. 264-273, 1991.

31 PREVIDI, M. P. Influenza del cloruro di sodio e della temperatura sullo sviluppo e sulla produzione di aflatossine da parte di Aspergillus parasiticus. Industria Conserve, v. 62, p. 215-219, 1987.

32 PURCHASE, I. F. H.; STEYN. M. Extration and estimation of aflatoxin $\mathrm{M}_{1}$ in milk by processing. Food Cosmetic. Toxicol., v.10, p. 383-387, 1972.

33 REDIN, C. L. C.; HARRISON, M. A.; KVIEN, C. K. Aspergillus parasiticus growth and aflatoxin synthesis on florunner peanuts grown in Gypsum-supplemented soil. J. Food Protect., v. 56, n. 7, p. 59-594, jul. 1993. 
34 RUSUL. G.; EL-GAZZAR, F. E.; MARTH, E. H. Growth and aflatoxin production by Aspergillus parasiticus NRRL 2999 in the presence of acetic or propionic acid and at different initial $\mathrm{pH}$ values. $\mathbf{J}$. Food Protect., v. 50, n.11, p. 909-914, Nov., 1987.

35 SANDERS, T. H.; DAVIS, N. D.; DIENER, U. L. Effect of carbon dioxide, temperature, and relative humidity on production of aflatoxin in peanuts. Journal of the American Oil Chemists Society, v. 45, n. 10, p. 683-685, 1968.

36 SCHINDLER, A. F.; PALMER, J. G.; EISENBERG, W. V. Aflatoxin production by Aspergillus flavus as related to various temperatures. Applied Microbiology, v. 15, n. 5, p. 1006-1009, 1967.

37 SCUSSEL, M. V. Micotoxinas em alimentos. Florianópolis: Insular, 1998. 144p.

$38 \mathrm{SHIH}, \mathrm{C} . \mathrm{N}$; ; MARTH, E. H. Experimental production of aflatoxin on brick cheese. J. Milk Food Technol., v. 35, n. 10, p.109117, 1972.

39 SING, P. L.; AHMED, S. K.; BHAGAT, S. Effect of salinity on aflatoxin production by $A$. flavus. Indian Phytopathology, v.40, n. 4, p.553-555, 1987.

40 SORENSON, W. G.; HESSELTINE, C. W.; SHOTWELL, C. L. Effect of temperature on production of aflatoxin on rice by Aspergillus flavus. Mycopathol. Mycol. Appl., v. 33, p. 49-55, 1967.

41 STOLOFF, L. et al. Stability of aflatoxin $\mathrm{M}_{1}$ in milk. J. Dairy Sci., v. 58, n. 12, p. 1789-1793, 1975.

42 TORREY, G. S.; MARTH, E. H. Temperature in home refrigerators and mold growth at refrigeration temperatures. J. Food Protect., v. 40, n. 6, p. 393-397, 1977.

43 TRABULSI, L. R. et. al. Biologia dos fungos. In: GOMPERTZ, O. F. et al. Microbiologia médica. São Paulo: Atheneu, 1999. p. $365-374$. 
44 WOOD, G. E. Mycotoxins in foods and feeds in the United States. Journal of Animal Science, v. 70, n. 12, p. 3941-3949, Dez. 1992. 